\title{
El estatus metateórico de ZFEL
}

\section{The meta-theoretical status of ZFEL}

\author{
Ariel Jonathan Roffé; Santiago Ginnobili** \\ *CEFHIC-UNQ-CONICET, Universidad de Buenos Aires, \\ Universidad Nacional de Tres de Febrero, Argentina \\ ariroffe@hotmail.com \\ ${ }^{* *}$ CEFHIC-UNQ-CONICET, Universidad de Buenos Aires, \\ Universidad Nacional de Tres de Febrero, Argentina \\ santi75@gmail.com
}

\begin{abstract}
Resumen
En un libro reciente McShea y Brandon defienden que la diversidad y la complejidad de la vida se explican, principalmente, por la acción de un principio que llaman "la ley evolutiva de fuerzas cero" o "ZFEL". Tal principio actuaría de un modo implícito por detrás de muchas explicaciones de la biología, pero nunca habría sido explicitado. Asumiendo que esta idea es interesante, y que los autores en cuestión tienen razón, discutiremos el modo metateórico en que presentan dicho principio, como siendo parte de la teoría de la probabilidad. Esto permite a los autores afirmar que la teoría de la probabilidad brindaría la base reductiva para toda la biología evolutiva (dado que consideran que otros principios, como el de selección natural, también serían parte de la teoría de la probabilidad). Defenderemos que, efectivamente, ZFEL no es propio de la biología únicamente, pero no por formar parte de la teoría de la probabilidad, sino por tratarse de una versión específica del principio de causa común.
\end{abstract}

Palabras clave: ley evolutiva de fuerzas cero, complejidad, diversidad, teoría de la probabilidad, principio de causa común.

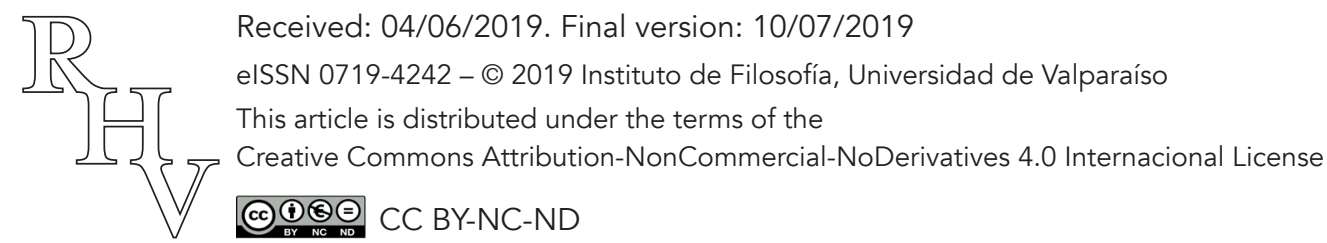




\begin{abstract}
In a recent book, McShea and Brandon argue that the observed diversity and complexity of life are explainable by a principle they call the "zero-force evolutionary law" or "ZFEL". Although this principle would be implicit in many explanations given by biologists, it would have never been made explicit. Assuming that this idea is interesting, and that the authors are right, we will discuss the metatheoretical way in which they present said principle, as being a part of probability theory. This allows the authors to claim that probability theory provides the reductive basis for all evolutionary biology (given that they consider other principles, such as the principle of natural selection, as part of probability theory as well). We will defend, in accordance with them, that ZFEL is not a solely biological principle, but not because it is a part of probability theory, but rather because it is a specific version of the principle of common cause.
\end{abstract}

Keywords: zero-force evolutionary law, complexity, diversity, probability theory, principle of common cause.

\title{
1. Introducción
}

Entre los enigmas fundamentales que Darwin intentó desentrañar en su obra monumental, se encuentra el dar cuenta de la diversidad de la vida. La respuesta explicita que Darwin da a esta cuestión apela al principio de divergencia, que (dejando de lado discusiones acerca de su naturaleza, alcance o adecuación, p.e. Richards 2012) parece vincularse intrínsecamente con la teoría de la selección natural.

Según tal principio,

Los descendientes modificados de cualquier especie prosperarán tanto mejor cuanto más diferentes lleguen a ser en su conformación y sean de este modo capaces de usurpar los puestos ocupados por otros seres. (Darwin 1859, 116)

Darwin considera que la selección natural produce diversidad, porque resulta una estrategia exitosa apartarse de la media de la especie y (dicho en lenguaje posterior) ocupar nuevos nichos en los que la competencia no sea tan feroz. Tal principio, según el mismo Darwin afirma, permitiría explicar la tendencia de los organismos a divergir de sus ancestros. El problema es caracterizado como de gran importancia, y el principio es presentado como la solución única y suficiente (Darwin 1958, 120-21).

Por supuesto, los científicos innovadores no suelen tener una comprensión total ni de la naturaleza y ni del alcance de su propia obra, y no debiera sorprendernos que Darwin, por carecer de las herramientas metateóricas y la comprensión de la ciencia que se ganaría en el siglo siguiente, no pudiera realizar exitosamente el salto semántico que involucra la presentación metateóricamente adecuada de las propias teorías y los vínculos interteó- 
ricos involucrados (sin dejar de señalar que Darwin es un autor con extrema claridad de pensamiento y en parte en eso debe haber radicado su éxito). Sin embargo, resulta algo sorprendente que en su ejemplar paradigmático de cómo nuevas especies divergen tanto de sus ancestros al punto de convertirse en nuevas especies, el caso de los pinzones en Galápagos, el principio de divergencia no juegue ningún rol.

La explicación darwiniana de la divergencia entre los diferentes pinzones apela al aislamiento geográfico de las islas con el continente y entre sí. Los pinzones habrían colonizado las islas gracias a medios ocasionales de transporte, y se habrían adaptado a sus diferentes ambientes (Darwin 1859, 400-401). Por supuesto, el mecanismo principal por el cual habría ocurrido la adaptación, según Darwin, habría sido la selección natural (Darwin 1859, 401), pero no a través del principio de divergencia, sino, sencillamente, por los diferentes caminos evolutivos que se producirían gracias a los diferentes nichos existentes en las diferentes islas.

Este desfasaje existente entre las explicaciones brindadas por Darwin, y su propia presentación y comprensión metateórica de sus propias ideas, permite realizar una introducción adecuada al trabajo de McShea y Brandon en el que intentan explicitar y elucidar el principio que en la biología actual, según afirman, explica en mayor grado la diversidad y la complejidad de los organismos vivos, aunque funcionando de manera implícita (McShea y Brandon 2010). Como ocurría con Darwin, el modo en que los biólogos contemporáneos explican la diversidad (y también la complejidad) de los organismos vivos, y el modo en que creen que lo hacen, no coincide. Podemos, de hecho, encontrar la misma tensión que existe en Darwin, a la hora de explicar la diversidad, en manuales de biología evolutiva. Por ejemplo Ridley (2004, 487-89), explica el patrón jerárquico de la filogenia y la forma arbórea de la evolución, apelando al principio de divergencia darwiniano. Pero al igual que veíamos en el caso de Darwin, el principio de divergencia no cumple ningún rol a la hora de explicar la especiación en el caso de los pinzones de Galápagos (Ridley 2004, 595-96). Otra vez, la selección natural operando de manera independiente en cada isla, parece ser suficiente.

La principal meta del trabajo de McShea y Brandon consiste en la correcta enunciación del principio que explica la complejidad y diversidad de los organismos vivos en mayor medida, que funciona implícitamente unificando muchas áreas y explicaciones de la biología evolutiva: la ley evolutiva de fuerzas cero (en adelante ZFEL, por sus siglas en inglés). Presentaremos ZFEL en la sección 2 del trabajo.

McShea y Brandon defienden tres tesis de diferente tenor:

1. ZFEL es un principio que opera implícitamente en la biología actual y por lo tanto merece ser explicitado y reconstruido.

2. ZFEL logra explicar la diversidad y la complejidad de manera más adecuada o en mayor grado que otros mecanismos que pretenden explicarlas. 
3. Finalmente, los autores hacen una serie de afirmaciones acerca de la naturaleza metateórica de ZFEL, que se trata de una ley universal, que funciona como hipótesis nula, que puede ser pensando, en analogía con la mecánica clásica, como una ley de fuerzas cero. Sin embargo, la afirmación más sustantiva de las que realizan los autores tiene que ver con que ZFEL es un principio que forma parte de la teoría de la probabilidad.

En este trabajo no discutiremos los primeros dos puntos, que nos parecen bien defendidos por los autores ${ }^{1}$, sino que nos concentraremos en la discusión acerca de la naturaleza metateórica del principio. En particular con su idea de que ZFEL no es más que una parte de la teoría de la probabilidad aplicada a la biología, que sumada a su idea de que el principio de selección natural también es una parte de la teoría de la probabilidad aplicada, los lleva a sostener que la teoría de la probabilidad es la base reductiva de la biología evolutiva (McShea y Brandon 2010, 108-9). Presentaremos esta idea en la sección 3.

En este trabajo defenderemos que, si bien es cierto que puede utilizarse la teoría de la probabilidad para presentar ZFEL, no es adecuado considerar que tal principio se identifica con una parte de la teoría de la probabilidad. Pues, como intentaremos mostrar en la sección 4, la naturaleza transdisciplinar de ZFEL (o de una versión más generalizada de ZFEL), señalada por los mismos autores, queda mejor reflejada si se considera a ZFEL como una aplicación del principio de causa común (PCC en adelante) a la biología, y, como sostendremos, no puede considerarse a tal principio como reducible a la teoría de la probabilidad. Si bien no es tema de este trabajo discutir la naturaleza del principio de causa común, presentaremos en la sección 5 una breve reseña de la discusión respecto a la naturaleza del tal principio. En la sección 6 estarán nuestras conclusiones.

\section{La ZFEL}

En su libro Biology's First Law, McShea y Brandon introducen un principio que, según ellos, los biólogos evolutivos utilizan implícitamente para explicar la gran diversidad y complejidad de la vida. Llaman a este principio ZFEL (por Zero-Force Evolutionary Law) y lo enuncian del siguiente modo: "En cualquier sistema evolutivo en el que hay variación azarosa y herencia, en ausencia de fuerzas y constricciones, la diversidad y la complejidad tenderán a aumentar" (McShea y Brandon 2010, 4).

Es necesario hacer una serie de clarificaciones para ver cómo estos autores comprenden este principio y sus alcances. En primer lugar, según ellos, la ZFEL se aplica de manera transversal a muchos niveles simultáneamente (McShea y Brandon 2010, 9-10). Por ejemplo, si cada población dentro de un ensamble de poblaciones evoluciona inde-

\footnotetext{
${ }^{1}$ Para discusiones respecto a la enunciación de ZFEL y su importancia y alcance puede apelarse a (Barrett et al. 2012), así como a la respuesta que los autores dan en (Brandon y McShea 2012).
} 
pendientemente, la expectativa sería la dispersión / el aumento de la varianza (medida genotípica o fenotípicamente) en el nivel del ensamble. Lo mismo ocurrirá con un conjunto de especies dentro de un género, un conjunto de géneros dentro de una familia o incluso un conjunto de órganos dentro de un organismo (a través de las generaciones). Una analogía ilustrativa propuesta por los mismos autores para entender la idea general es la de un conjunto de personas que caminan en diferentes direcciones, independientemente unas de otras. En ausencia de constricciones que los hagan caminar hacia el mismo lugar, la expectativa es que las personas se alejen más y más unas de otras.

En segundo lugar, los mecanismos mediante los cuales los individuos del ensamble (en el nivel que sea) evolucionan pueden ser de distinto tipo - i.e. el requisito de evolución independiente puede instanciarse de diversos modos. En particular, en los casos biológicos de interés, los individuos del ensamble (que recuérdese, pueden ser poblaciones / especies / etc.) pueden estar evolucionando o bien por deriva o bien por selección natural. En el primer caso, la dispersión se dará porque la deriva es evolución azarosa, lo cual significa que los individuos no tenderán a ir hacia un punto común. En el segundo caso, sólo se cumplirá el requisito de ausencia de constricciones cuando los óptimos hacia los cuales la selección hace tender a los individuos del ensamble sean independientes unos de otros (lo cual, a su vez, puede entenderse como presiones selectivas no compartidas). Un ejemplo del segundo tipo de caso sería el ya mencionado de los pinzones de Galápagos. Dado que las presiones selectivas a las que están sometidas las poblaciones de pinzones de cada una de las islas son independientes entre sí (porque cada isla plantea problemas ambientales diferentes), la ZFEL indica que las poblaciones de pinzones tenderán a alejarse entre sí, como de hecho ocurrió. Volviendo al ejemplo de los caminantes, la dispersión entre ellos puede ser resultado de que caminan en direcciones azarosas (deriva) o de que cada una camina en una dirección determinada, no azarosa, - por ejemplo, una camina hacia su casa, otra hacia su trabajo, etc. - pero que no haya ninguna constricción que los haga caminar hacia el mismo lado (selección).

A su vez, nótese que un mecanismo evolutivo puede reducir la diversidad (fenotípica o genotípica) en un nivel y aumentarla en otro. Por ejemplo, tanto la selección direccional como la deriva tienden a reducir la diversidad a nivel intrapoblacional, pero pueden aumentarla interpoblacionalmente, si diferentes poblaciones fijan distintos rasgos o genes. Esto ilustra nuevamente el hecho de que la ZFEL se aplica en distintos niveles independientemente unos de otros. Adicionalmente, el hecho de que los mecanismos que originan la diversidad en el nivel superior puedan ser de diversos tipos otorga a ZFEL un gran poder unificador, mostrando que fenómenos en apariencia muy distintos (p.e. la especiación alopátrica y la acumulación de variaciones azarosas en regiones no codificantes del genoma) explican el surgimiento de la diversidad de modos similares.

En tercer lugar, es necesario aclarar cómo se relacionan la diversidad y la complejidad para McShea y Brandon, ya que la ZFEL predice un aumento de ambas. Al hablar de complejidad los autores no pretenden hablar del uso coloquial de dicho concepto, 
que suele involucrar cosas como funcionalidad, sofisticación, etc. En cambio, utilizan un concepto que llaman "complejidad pura", definido únicamente a partir de la cantidad de tipos de partes que tiene una entidad, o el grado de diferenciación entre ellas (McShea y Brandon 2010, 7, 46), sean funcionales o no. De este modo, la complejidad en un nivel no es más que la diversidad vista desde un nivel superior. Por ejemplo, la complejidad de un género es la diversidad de las especies que lo componen; la complejidad de la columna vertebral es la diversidad -como cantidad de tipos- de vertebras que contiene (McShea y Brandon 2010, 10).

Un último punto aclaratorio refiere a lo que los autores entienden por constricciones y fuerzas. En este punto, McShea y Brandon siguen a Sober (1984), quien presentó a la teoría evolutiva como una teoría de fuerzas (análoga a la mecánica clásica newtoniana). ${ }^{2}$ En dicho libro, Sober presenta a la teoría evolutiva como estando caracterizada por una ley de fuerzas cero, que especifica el estado en el que se encontrará una entidad en ausencia de fuerzas, y un conjunto de fuerzas que pueden alejar a las entidades de ese estado, y que pueden componerse entre sí para actuar conjuntamente. Partiendo del modo como la genética de poblaciones caracteriza a los procesos microevolutivos, Sober había identificado a la ley de fuerzas cero con la ley Hardy-Weinberg, y por consiguiente a la mutación, migración, deriva y selección como las fuerzas relevantes. En consecuencia, para este autor, la expectativa en ausencia de fuerzas es la estasis, siendo el cambio el que debe ser explicado postulando fuerzas.

La posición de McShea y Brandon puede verse como una aceptación del marco general de la analogía, pero discutiendo cuáles son las fuerzas, y por consiguiente el estado libre de fuerzas. Uno de los objetivos centrales de estos autores es provocar un cambio gestáltico, redibujando el panorama conceptual sobre qué es lo esperable y qué es lo que requiere una explicación especial. Contrariamente a Sober, sostienen que lo esperable es el cambio, y que la estasis / la estabilidad es lo sorprendente, y es algo que requiere una explicación que apele a algún tipo de factor adicional, fuerza o constricción. La clave para operar este cambio consiste en no considerar como fuerzas / constricciones ni a la mutación ni a la deriva. Por consiguiente, una población evolucionando en ausencia de fuerzas estará sometida a la mutación y a la deriva, y por consiguiente tenderá a variar.

McShea y Brandon ofrecen diferentes motivos para no considerar a la mutación y la deriva como fuerzas. Por ejemplo, respecto de la deriva, afirman que no es analogable a una fuerza porque no tiene una dirección predecible a priori (vease también Brandon 2006; Roffé 2017). A su vez, tanto de la mutación como de la deriva, afirman que no son fuerzas porque son "constitutivas" de los sistemas evolutivos. Con esto quieren decir que deben ser relegadas al background explicativo, o bien porque son necesarias o bien por

\footnotetext{
${ }^{2}$ Sober no fue el primero ni el último en trazar esta analogía. Sin embargo, Sober sí fue quien articuló la analogía en detalle por primera vez, dando origen a una amplia literatura en filosofía de la biología al respecto.
} 
motivos heurísticos (ambos puntos les fueron discutidos, véase Luque 2016; Pence 2017). No nos ocuparemos aquí de examinar la adecuación de los motivos que ofrecen para defender estos puntos. En la sección siguiente nos centraremos una característica adicional que le atribuyen a ZFEL, y que será el foco central de nuestro análisis: el hecho de no ser más que teoría de la probabilidad aplicada.

\section{ZFEL y la teoría de la probabilidad}

En esta sección nos enfocamos en el modo en que ZFEL es presentado por McShea y Brandon. Concediendo que efectivamente, ZFEL parece funcionar implícitamente en diferentes partes de la biología, discutiremos el modo en que es explicitado por los autores, especialmente, la idea que proponen de que ZFEL se derivaría de la teoría de la probabilidad:

ZFEL surge de propiedades contingentes de la variación en la naturaleza, propiedades que son del dominio formal de la teoría de la probabilidad. (McShea y Brandon 2010, 7)

Más bien, para ZFEL, como para PNS [el principio de selección natural], la teoría de la probabilidad provee la fundación reductiva para la teoría evolutiva universal [...] la ZFEL se sigue deductivamente de la teoría de la probabilidad. (McShea y Brandon 2010, 109)

La razón que brindan para sostener esto se encuentra fundamentalmente en el capítulo 2 del libro, y se relaciona con su "modelo de la partícula", de modo que es necesario presentar este modelo abstracto, así como elucidar por qué los autores creen que el modelo tiene algún tipo de relevancia para la biología.

El modelo consta de una partícula que se mueve azarosamente (con probabilidad 0,5 ) o bien un paso a la izquierda, o bien un paso a la derecha (no puede permanecer en el mismo lugar) en cada momento. Si representamos cada momento en un eje vertical, la distribución de probabilidades para el movimiento de la partícula tras cuatro momentos se ve como sigue: 
El estatus metateórico de ZFEL

Ariel Jonathan Roffé; Santiago Ginnobili

\begin{tabular}{|c|c|c|c|c|c|c|c|c|}
\hline 0,0625 & & $\mathbf{0 , 2 5}$ & & $\mathbf{0 , 3 7 5}$ & & $\mathbf{0 , 2 5}$ & & 0,0625 \\
\hline & 0,125 & & 0,375 & & 0,375 & & 0,125 & \\
\hline & & 0,25 & & 0,5 & & 0,25 & & \\
\hline & & & 0,5 & & 0,5 & & & \\
\hline 0 & & 0,25 & & 0,5 & & 0,75 & & 1 \\
\hline
\end{tabular}

Fig. 1. Distribución de probabilidad para el modelo de la partícula de McShea y Brandon.

Lo que el modelo de la partícula representa gráficamente es un proceso de muestreo de Bernoulli. Este tipo de proceso es uno en el cual una muestra de tamaño $n$ es extraída, con reemplazo, de una población compuesta de dos tipos de objetos.

Por ejemplo, puede pensarse a la población como una urna que contiene un conjunto de bolillas, las cuales son de dos colores (p.e. rojo y azul). Supóngase que hay un número infinito de bolillas en la urna, que la frecuencia $p$ de bolillas rojas es 0,5 (i.e. la mitad son rojas y la otra mitad azules), que el tamaño $n$ de muestra es 4 . La probabilidad de que la muestra contenga $k=4$ bolillas rojas puede calcularse del siguiente modo. La probabilidad de que la primera bolilla muestreada sea roja es 0,5 . Del $50 \%$ de los casos en los que la primera bolilla es roja, habrá otro $50 \%$ de casos en donde la segunda también será roja. Esto es, la probabilidad de que las primeras dos bolillas muestreadas sean rojas es $0,5 \times$ $0,5=0,25$ (nótese que este es el número de la derecha en el momento $s_{2}$, en la Fig. 1). Utilizando un razonamiento análogo, puede notarse que la probabilidad de que la muestra contenga a las 4 bolillas rojas es $0,5^{4}=0,0625$ (nuevamente, véase el valor de la derecha en la fila $s_{4}$, Fig. 1). El cálculo para $k$ distintos de 0 y 4 es un tanto más complejo. Por ejemplo, existen cuatro modos posibles de obtener una muestra con $k=1$ bolillas rojas, que corresponden con las cuatro secuencias $\langle R, B, B, B\rangle,\langle B, R, B, B\rangle,\langle B, B, R, B\rangle, y\langle B, B,-$ $\mathrm{B}, \mathrm{R}\rangle$, cada una de las cuales tiene una probabilidad de $0,5^{1} \times 0,5^{3}$. El resultado es, por tanto, 0,25 (véase la segunda columna de la fila $s_{4}$ ). De manera más general, la probabilidad de que la muestra contenga exactamente $k$ bolillas rojas y $n-k$ azules está dado por:

$$
\left(\begin{array}{l}
n \\
k
\end{array}\right) p^{k} q^{n-k}
$$

lo cual es conocido como una distribución binomial. 
En el gráfico de la partícula, muestrear un individuo rojo estaría representado por un movimiento de la partícula hacia la derecha, mientras que uno azul lo estaría con un movimiento hacia la izquierda (la probabilidad de ir a la derecha y a la izquierda representarían la frecuencia de las bolillas rojas y azules en la población). Así, el tamaño de la muestra está representado por la cantidad de filas. Los diferentes modos de obtener una muestra con la misma composición (es decir, con una distribución dada de frecuencias) están representados en el gráfico de la partícula como distintos caminos llevando al mismo punto. Así, en la fila superior, el gráfico de la partícula contiene los mismos valores que una distribución binomial.

Un motivo para pensar que este modelo matemático abstracto ilustra algo acerca de poblaciones biológicas es que la genética de poblaciones suele representar a las transiciones generacionales en términos de procesos de muestreo (Beatty 1984, 188-189, los llama "muestreo parental" y "muestreo de gametas"). De ese modo, el modelo de la partícula puede verse como una representación de los procesos probabilísticos usados en genética de poblaciones para inferir la composición genética de las generaciones filiales. Cada partícula que evoluciona independientemente representaría entonces una población biológica. Dada la distribución de probabilidades ilustrada en la Fig. 1, el modelo ilustraría así que un conjunto de poblaciones que parten de un mismo punto, si son dejadas a evolucionar independientemente, tenderán a alejarse entre sí (i.e. la varianza para el parámetro $p$ aumenta entre ellas de $s_{1}$ a $s_{4}$ ). Los autores parecen ver a esto como una demostración matemática de ZFEL.

Nuevamente, una serie de autores han objetado a los alcances de esta demostración. Por ejemplo, Barret et al. (2012) y Roffé (2017) notan que si las poblaciones fuesen iniciadas en puntos distintos del espacio (p.e. todas muy cerca de $p=1$ ) entonces, dado que la deriva tiende a fijar el alelo más frecuente, la varianza entre ellas tendería a reducirse, no a aumentar (es decir, el resultado dependería de unas condiciones iniciales particulares, no valiendo en otras). Del mismo modo, el modelo de la partícula presentado en la Fig. 1 representa solamente una transición generacional. Pero la genética de poblaciones estándar muestra que, en el largo plazo, si las poblaciones evolucionan independientemente por deriva, entonces todas tenderán a fijar o bien uno o bien el otro alelo (i.e. las poblaciones se acumularían todas en los dos extremos de la distribución, tras lo cual la diversidad se estancaría).

En su respuesta, McShea y Brandon afirman que ambos casos de estancamiento o reducción de la varianza se deben a que hay una constricción operando: el hecho de que haya "barreras absorbentes" en los extremos de la distribución -i.e. el hecho de que una frecuencia no pueda ir más allá de 0 o 1 . Es decir, dado que la posición que ocupa la partícula representa una frecuencia, el "espacio" en el que esta puede moverse es finito. Pero si esta constricción no estuviese presente, las partículas seguirían alejándose entre sí incluso en el largo plazo (McShea y Brandon 2010, 104; Brandon y McShea 2012, 741). 
Más recientemente, los autores presentaron una generalización del aparato matemático mediante el cual supuestamente "demostraron" ZFEL (McShea, Wang, y Brandon 2019). En este trabajo, las partículas pueden no solo moverse hacia la izquierda o la derecha, sino también mantenerse en el lugar (i.e. ir hacia arriba en el espacio). Adicionalmente, no suponen en este trabajo que las partículas comienzan todas en el mismo lugar, sino que asumen que ellas comienzan a cualquier distancia arbitraria. El modo de demostrar el resultado consiste en obtener las probabilidades de que la distancia entre ellas se mantenga idéntica tras $n$ generaciones (estasis y cambio paralelo), de que se reduzca (llaman a esto cambio convergente) y de que aumente (cambio divergente). McShea, Wang y Brandon muestran que, en el largo plazo, la probabilidad de cambio divergente es mayor a la probabilidad de cambio convergente y de que no haya cambio, y que la distancia esperada entre dos partículas tiende a ir en aumento. En el caso extremo en que dos partículas se encuentran inicialmente a cierta distancia, y que la de la izquierda tiene mayor probabilidad de ir hacia la derecha y viceversa, si bien la distancia entre ellas se reducirá inicialmente, con el paso de las generaciones volverá a aumentar dado que las trayectorias se cruzarán. Nuevamente, sin embargo, la demostración asume que el espacio no tiene barreras absorbentes, y que las probabilidades para el movimiento de las partículas no cambian según la región del espacio en la que se encuentran. Por ese motivo, es lícito preguntarse si responden completamente a las objeciones planteadas anteriormente.

Nuevamente, no nos ocuparemos de examinar la adecuación de estas objeciones, ni de las respuestas. En la sección siguiente, plantearemos un tipo de objeción distinta, no tanto al contenido fáctico de ZFEL sino al estatus metateórico (de ser un resultado puramente probabilístico) que McShea y Brandon le atribuyen.

\section{ZFEL y el principio de causa común}

La presentación de McShea y Brandon de ZFEL adecuada a los fines de explicar con claridad en qué consiste el principio y cuál es su importancia. Sin embargo, puede preguntarse, por un lado, si esta presentación en términos de probabilidad pura es suficiente para caracterizar el fenómeno biológico de interés, o si la aplicación a la biología presupone otros principios fácticos. Por otro lado, cabe cuestionarse si la presentación en términos probabilísticos es necesaria, o si el principio puede ser presentado de otros modos. Si bien creemos que la respuesta a ambas preguntas es negativa, en lo que sigue, nos centraremos en la segunda. En esta sección, asumiendo que ZFEL puede presentarse adecuadamente apelando a conceptos probabilísticos, defenderemos que ello no es necesario. Argumentaremos que ZFEL es una instancia o especificación de un principio mucho más general, que puede aplicarse transversalmente a una gran cantidad de disciplinas científicas: el PCC. Tal principio, que puede y ha sido elucidado a través de nociones probabilísticas, trasciende la teoría de la probabilidad. 
Un primer argumento de plausibilidad para sostener la tesis de que la teoría de la probabilidad no es necesaria para la utilización de ZFEL es que, según los propios autores, esta ley está presupuesta en eventos de especiación alopátrica. Como dijimos en la sección I, el propio Darwin apela a este tipo de especiación en sus textos (p.e. en casos como el de los pinzones). Darwin, sin embargo, apela a este tipo de especiación sin utilizar conceptos probabilísticos. En cambio, puede afirmarse que está utilizando una versión más potente de ZFEL, no probabilística, que queda mejor presentada en términos causales como una versión del PCC. Como veremos a continuación, la presentación de ZFEL en términos del PCC permite ver que, tanto las explicaciones cuantitativas de la genética de poblaciones (ya que el PCC puede elucidarse probabilísticamente) como las más cualitativas (tanto darwinianas como de especiación alopátrica en exposiciones actuales), instancian un mismo patrón explicativo.

Detrás de la idea de que la evolución independiente en las diferentes islas lleva a la diversificación, lo que se encuentra presupuesto (por fuera de todo el lenguaje estadístico / probabilístico de la genética de poblaciones) es un principio condicional como el siguiente:

No hay causas comunes operando sobre la diversidad en dos $\longrightarrow \quad \begin{aligned} & \text { La diversidad entre ambas poblaciones } \\ & \text { se incrementará }\end{aligned}$
poblaciones diferentes poblaciones diferentes

A su vez, el condicional de arriba es equivalente a:

No hay causas comunes operando

sobre la diversidad en dos

poblaciones diferentes

No es cierto que el curso evolutivo de ambas poblaciones coincidirá

Como se vio, el motivo por el que el consecuente vale dado el antecedente es que si las poblaciones realmente evolucionan independientemente (i.e. si no hay causas comunes), entonces que su curso evolutivo coincida sería una coincidencia altamente improbable. ZFEL puede pensarse entonces como una versión más específica y en el dominio de la evolución de un principio más general que afirma que:

No hay causas comunes

$\longrightarrow \quad$ No ocurre una coincidencia improbable

La contrapositiva de este condicional afirma que:

Ocurre una coincidencia impobrable $\longrightarrow$ Hay una causa común operando 
Lo cual no es otra cosa que el PCC. En su formulación estándar, el PCC afirma justamente "Si una coincidencia improbable ha ocurrido, entonces debe existir una causa común" (Reichenbach 1956, 157). Para que la coincidencia sea improbable, por supuesto, debe existir una correlación sin una relación causal directa entre los eventos para los que se da la coincidencia. En la versión de Sober, también informal y apenas más sofisticada, se incluye esta cuestión "Si el evento X e Y correlacionan, entonces o X causó Y, o Y causó X, o X e Y son efectos conjuntos de una causa común” (Sober 2001, 331).

Puede objetársenos que la formulación del antecedente del primer condicional presentado presupone parte de lo que queremos mostrar (ya que está formulada en términos de causas comunes y no de fuerzas o constricciones). Esta objeción, a su vez, puede dividirse en dos. En primer lugar, puede formularse la objeción al uso del vocabulario causal. Existe un largo debate acerca de si los análogos a las fuerzas newtonianas en la teoría evolutiva pueden o no ser pensados como causas (p.e. Baravalle y Vecchi, por aparecer; Matthen y Ariew 2002; Stephens 2004; Lewens 2010; Reisman y Forber 2005; Walsh, Lewins, y Ariew 2002). Sin embargo, esta discusión va más allá de nuestro punto. Podrían aceptarse ZFEL y el uso del PCC en la biología sin comprometerse con la causalidad en un sentido sustantivo (p.e. los propios empiristas lógicos tenían reservas con el uso del vocabulario causal, y sin embargo se dedicaron a elucidar el PCC). En todo caso, la discusión pasaría por ver cómo entender al concepto de causa del PCC.

Por otro lado, incluso concediendo que las fuerzas evolutivas son causas, podría objetarse la formulación en términos de causas comunes. Es decir, en su formulación original, ZFEL vale en ausencia de fuerzas y constricciones. Si se acepta hablar de causas en lugar de fuerzas y constricciones, esto equivale a hablar de ausencia de causas, no de causas comunes. Sin embargo, la formulación original de ZFEL sí asume que las fuerzas y constricciones son compartidas. Por ejemplo, como se vio arriba, la selección natural sólo va en contra de ZFEL (i.e. solo se incumple la condición de ausencia de fuerzas) cuando las presiones selectivas son compartidas. Caso contrario (presiones selectivas independientes) el antecedente de ZFEL vale. Por ese motivo, no creemos que sea distorsionador hablar en términos de causas comunes.

Otras aplicaciones (no biológicas) del PCC pueden ilustrar su similaridad con ZFEL. Por ejemplo, el siglo XVI, cuando se descubrió una nova que no cambiaba su posición relativa con las estrellas fijas, obligaba a los que defendían el modelo aristotélico a ubicarla sobre el mismo soporte que al resto de las estrellas fijas. Esta es la razón por la cual la nova tenía un carácter anómalo para la astronomía antigua en la que no podían ocurrir cambios en el mundo supralunar. En este caso, la aplicación del principio en juego sería: Si la posición de un objeto celeste con respecto a las estrellas fijas no cambia (lo cual constituiría una coincidencia improbable) entonces el objeto está engarzado en la misma esfera que ellas (la causa común de su movimiento en el cielo). Al igual que con ZFEL, podría presentarse este principio aplicado por la contrapositiva: si la trayectoria de un 
objeto celeste no mantiene su posición relativa con las estrellas fijas (i.e. si no ocurre una coincidencia improbable), entonces no está engarzado en la esfera correspondiente (no hay una causa común).

Qué cuenta como causa común depende del tipo de causas que gobiernan los fenómenos en cuestión (o del marco conceptual con el que uno investigue el tipo de fenómenos en cuestión). Lo que vuelve específico al PCC en su aplicación a la biología (i.e. ZFEL) tiene que ver con el tipo de factores que pueden operar como causas comunes: migración, selección natural estabilizadora, mismas presiones selectivas, etc., y a lo que constituiría una coincidencia improbable: la coincidencia en el camino evolutivo específico.

Antes de proseguir, es necesario hacer una aclaración importante. En el caso biológico, si dos poblaciones coinciden en su trayectoria evolutiva, esto puede deberse o bien a una causa común o bien a un tipo de causa común. Por ejemplo, si dos poblaciones distintas de polillas en un mismo bosque evolucionan un patrón de coloración oscuro porque la polución industrial contamina los árboles del bosque, entonces estaríamos ante un caso de una única causa común. Sin embargo, si dos poblaciones en dos bosques distintos evolucionan la coloración oscura porque ambos bosques fueron contaminados por distintas industrias, entonces estamos ante un caso de un mismo tipo de causa (y la explicación apela a algo así como un PCC para tipos). Esto, que puede implicar algunos detalles en la discusión acerca del modo más adecuado de pensar la naturaleza del PCC (ver por ejemplo, Sober 2001), para el punto de nuestro trabajo no es difícil de conceder. Piénsese el ejemplo brindado por el mismo Reichenbach de aplicación del PCC en la vida cotidiana: el caso en que dos lámparas se apagan a la vez (Reichenbach 1956, 157). Sería una causa común que la misma causa específica actúe, p.e. un corte de luz, pero también lo sería que ambas lámparas tuvieran la misma fecha de expiración. Este segundo caso sería una causa común en el mismo sentido en que la convergencia como adaptación a mismos ambientes lo es. Esto nos basta para continuar. En adelante, haremos omisión de este importante detalle, no diferenciando entre un PCC para casos y otro para tipos.

\section{El principio de causa común y la teoría de la probabilidad}

Entender que ZFEL es una instancia del PCC es aclarador respecto de varios puntos. Por ejemplo, McShea y Brandon defienden la naturaleza transdisciplinar de una versión generalizada de ZFEL, que denominan G-ZFEL: "en un sistema en el cual hay persistencia, variación y memoria, en la ausencia de fuerzas y constricciones actuando sobre la diversidad y la complejidad, la diversidad y la complejidad aumentarán en promedio" (McShea y Brandon 2010, 111-12) Según los autores, esto aplicaría tanto a poblaciones biológicas de individuos (en donde persistencia y memoria son entendidos como reproducción y herencia) como a las estacas individuales de una cerca (en donde persistencia y memoria son continuidad a través del tiempo y perpetuación de los accidentes que ocurran a cada estaca — p.e. no hay nadie pintando la cerca continuamente). Es decir, dada

Revista de Humanidades de Valparaíso, 2019, No 14, 57-73

(c) (1) @ $\odot$ CC BY-NC-ND 
una cerca inicialmente igual, si cada estaca es víctima de accidentes diferentes, la cerca se volverá más compleja (las estacas más diversas). Que ZFEL (o en este caso G-ZFEL) sean instancias del PCC explica su naturaleza transdisciplinar.

Respecto del punto que es objetivo de este artículo, que es entender la relación entre ZFEL y la teoría de la probabilidad, la subsunción de ZFEL bajo el PCC también resulta esclarecedora. Las relaciones entre las diversas teorías de la causalidad y la teoría de la probabilidad son complejas. Pero en principio puede afirmarse, de manera no muy controvertida, que:

- Las nociones probabilísticas pueden permitir caracterizar el fenómeno de la causalidad, e incluso la inferencia a la causa común.

- Las inferencias probabilísticas nos pueden permitir detectar relaciones causales.

En ambos casos la apelación a la probabilidad tiene que ver con que las correlaciones causales no son perfectas. Por ejemplo, tratando a la causalidad de modo humeano, como la mayoría de las veces la conjunción constante sobre la que la causalidad se sustenta es aproximada, es necesario caracterizarla apelando a nociones probabilísticas. Así, por ejemplo, Suppes define "causa" del siguiente modo (Suppes 1970, 12):

$B_{t}$ es prima facie causa del evento $A_{t}$ si y sólo si

(i) $t^{\prime}<t$,

(ii) $P\left(B_{\mathrm{t}}\right)>0$,

(iii) $P\left(A_{\mathrm{t}} / B_{\mathrm{t}}\right)>P\left(A_{\mathrm{t}}\right)$.

En el mismo sentido, en el caso del PCC, la coincidencia improbable puede ocurrir. Sólo que es improbable que ocurra. La inferencia a la causa común no es, en consecuencia, absolutamente certera, sino que aumenta de acuerdo a la improbabilidad de la coincidencia, o la repetición de las coincidencias (Reichenbach 1956, 158). Y esa es la razón por la que tal principio suele ponerse en términos probabilísticos. Por ejemplo, Van Fraassen $(1980,28)$ presenta el principio de Reichenbach del siguiente modo:

Si las coincidencias de dos eventos A y B ocurre más frecuentemente que lo que correspondería para su ocurrencia independiente, esto es, si los eventos satisfacen la relación

(1) $P(A \& B)>P(A) . P(B)$,

entonces debe existir una causa común $\mathrm{C}$ para esos eventos tal que la tríada $\mathrm{ACB}$ es conjuntiva, esto es, satisface las relaciones (2)-(5) siguientes:

(2) $P(A \& B / C)=P(A / C) \cdot P(B / C)$ 
(3) $P(A \& B / \bar{C})=P(A / \bar{C}) \cdot P(B / \bar{C})$

(4) $P(A / C)>P(A / \bar{C})$

(5) $P(B / C)>P(B / \bar{C})$

Independientemente de que este sea un modo de adecuado de dar cuenta del principio, lo que se puede afirmar con claridad es que el hecho de que se utilice teoría de la probabilidad para caracterizar la causalidad y el PCC o para detectar relaciones causales o causas comunes subyacentes, no implica que ni la causalidad ni el PCC (y por lo tanto ZFEL) sean parte de la teoría de la probabilidad. En el segundo caso (la utilización de la teoría de la probabilidad para detectar relaciones causales particulares) el punto es obvio. En el primer caso, porque la utilización de ciertos recursos matemáticos para formular una teoría fáctica no vuelve a la segunda parte de los primeros. De otro modo habría que decir que la mecánica clásica no es más que cálculo infinitesimal, que la astronomía antigua no es más que geometría, etc. La característica distintiva de las teorías fácticas (como lo sería una teoría de la causalidad a la Suppes, o una formulación del PCC a la Reichenbach) es que, si bien se formulan usando tales herramientas matemáticas, postulan axiomas nuevos e independientes por sobre los de la teoría formal que tienen a la base. Esto es, $F=m a$ no es un teorema del cálculo infinitesimal, así como tampoco lo son los axiomas con los que Suppes y Reichenbach caracterizan a la causalidad y el PCC de ningún cáclulo de probabilidad.

\section{Conclusiones}

En el presente trabajo, hemos presentado la ZFEL de McShea y Brandon - la ley que proponen como caracterizando el estado de fuerzas cero en la teoría evolutiva-, y discutido su estatus metateórico respecto a su relación con la teoría de la probabilidad.

Las primeras dos secciones mostraron que el modo en el que diversos autores y manuales dicen que explican la diversidad y la complejidad, y el modo como efectivamente lo hacen, no siempre coincide. En ese sentido, la ZFEL fue introducida como un principio que está presupuesto en las explicaciones que efectivamente se dan, y que es unificador respecto de un conjunto muy amplio de casos. Se presentaron además algunos detalles acerca del alcance y el significado de los términos de esta ley (sin entrar en la discusión acerca de ellos).

La tercera sección elucida el motivo por el cual los autores sostienen que la ZFEL no es más que teoría de la probabilidad aplicada. Se mostró por qué el modelo matemático abstracto, de una partícula que se mueve hacia los costados, sería relevante para comprender fenómenos biológicos. Ilustramos además por qué McShea y Brandon creen que la 
difusión que se observa en el modelo cuenta como una demostración de que la diversidad debe incrementarse en las poblaciones biológicas, y notamos algunas objeciones que se le han hecho a esta afirmación.

Por último, en las últimas dos secciones, examinamos la tesis metateórica según la cual ZFEL no es más que teoría de la probabilidad aplicada. Mostramos que el motivo por el cual esta ley es aplicable de un modo transdisciplinar tan amplio se debe a que no es otra cosa que la contrapositiva del PCC. Esta identificación nos permitió, además, en la sección $\mathrm{V}$, criticar a la tesis metateórica mencionada. Las relaciones entre la teoría de la probabilidad y la causalidad son complejas, pero en ningún caso nos permiten afirmar que las caracterizaciones de la causalidad en términos probabilísticos "reduzcan" a esta a pura probabilidad aplicada.

\section{Referencias bibliográficas}

Baravalle, L., Vecchi, D. (por aparecer). Drift as a Force of Evolution: A Manipulationist Account. Life and Evolution.

Barrett, M., Clatterbuck, H, Goldsby, N., Helgeson, C., McLoone, B., Pearce, T., Sober, E., Stern, R., Weinberger, N. (2012). Puzzles for ZFEL, McShea and Brandon's Zero Force Evolutionary Law. Biology and Philosophy, 27(5): 723-35.

Beatty, J. (1984). Chance and Natural Selection. Philosophy of Science, 51(2): 183-211. Retrieved from: http://links.jstor.org/sici?sici=0031-8248\%28198406\%2951\%3A2\%3C183\%3ACA $\mathrm{NS} \% 3 \mathrm{E} 2.0 . \mathrm{CO} \% 3 \mathrm{~B} 2-\mathrm{C}$

Brandon, R. N. (2006). The Principle of Drift: Biology's First Law. The Journal of Philosophy, 103(7): 319-35.

Brandon, R. N., McShea, D. W. (2012). Four Solutions for Four Puzzles. Biology and Philosophy, 27(5): 737-44.

Darwin, C. R. (1859). On the Origin of Species by Means of Natural Selection. London: John Murray.

Darwin, C. R. (1958). The Autobiography of Charles Darwin 1809-1882. With the Original Omissions Restored. Edited and with Appendix and Notes by His Grand-Daughter Nora Barlow. Edited by Nora Barlow. New York: W.W. Norton.

Fraassen, B. C. V. (1980). The Scientific Image. Oxford: Clarendon Press.

Lewens, T. (2010). The Natures of Selection. The British Journal for the Philosophy of Science, 61(2): 313-33. doi: https://doi.org/10.1093/bjps/axp041.

Luque, V. J. (2016). The Principle of Stasis: Why Drift Is Not a Zero-Cause Law. Studies in History and Philosophy of Science Part C: Studies in History and Philosophy of Biological and Biomedical Sciences, 57: 71-79. doi: https://doi.org/10.1016/J.SHPSC.2016.04.001 
Matthen, M., Ariew, A. (2002). Two Ways of Thinking about Fitness and Natural Selection. Journal of Philosophy, 99(2): 55-83.

McShea, D. W., Brandon, R. N. (2010). Biology's First Law. Chicago: The University of Chicago Press.

McShea, D. W., Wang, S. C., Brandon, R. N. (2019). A Quantitative Formulation of Biology's First Law. Evolution. doi: https://doi.org/10.1111/evo.13735

Pence, C. H. (2017). Is Genetic Drift a Force? Synthese, 194(6): 1967-88. doi: https://doi.org/10.1007/s11229-016-1031-2

Reichenbach, H. (1956). The Direction of Time. Berkeley: University of California Press.

Reisman, K., Forber, P. (2005). Manipulation and the Causes of Evolution. Philosophy of Science, 72(5): 1113-23. doi: https://doi.org/10.1086/508120

Richards, R. J. (2012). Darwin's Principles of Divergence and Natural Selection: Why Fodor Was Almost Right. Studies in History and Philosophy of Science Part C: Studies in History and Philosophy of Biological and Biomedical Sciences, 43(1): 256-68. doi: https://doi.org/10.1016/J.SHPSC.2011.10.014

Ridley, M. (2004). Evolution - Third Edition. Malden: Blackwell.

Roffé, A. J. (2017). Genetic Drift as a Directional Factor: Biasing Effects and a Priori Predictions. Biology \& Philosophy, 32(4): 535-58. doi: https://doi.org/10.1007/s10539-017-9575-1

Sober, E. (1984). The Nature of Selection. Chicago: The University of Chicago Press.

Sober, E. (2001). Venetian Sea Levels, British Bread Prices, and the Principle of the Common Cause. British Journal for the Philosophy of Science, 52(2): 331-46. doi: https://doi.org/10.1093/bjps/52.2.331

Stephens, C. (2004). Selection, Drift, and the 'Forces' of Evolution. Philosophy of Science, 71: 550-570.

Suppes, P. (1970). A Probabilistic Theory of Causality. Amsterdam: North-Holland Publishing Company.

Walsh, D., Lewins, T., Ariew, A. (2002). The Trials of Life: Natural Selection and Random Drift. Philosophy of Science, 69(3): 429-46. doi: https://doi.org/10.1086/342454 